\title{
Transfer functions for infinite-dimensional systems
}

\author{
Hans Zwart \\ Department of Applied Mathematics, University of Twente \\ P.O. Box 217, NL-7500 AE Enschede, The Netherlands \\ h.j.zwart@math.utwente.nl
}

May 7, 2004

\section{Introduction}

The notion of transfer functions is classical in systems theory. One could even state that without transfer functions there is no systems theory. Here we study transfer functions for the state linear system

$$
\begin{aligned}
\dot{x}(t) & =A x(t)+B u(t), \quad x(0)=x_{0} \\
y(t) & =C x(t)+D u(t),
\end{aligned}
$$

where $A$ is the infinitesimal generator of the $C_{0}$-semigroup $T(t)$ on the state space $X, B$ is a bounded linear operator from input space $U$ to $X, C$ is a bounded linear operator from $X$ to the output space $Y$, and $D$ is a bounded operator from $U$ to $Y$. The spaces $X, U$ and $Y$ are assumed to be Banach spaces. More detail on the system (1) can be found in Curtain and Zwart [1]. For the system (1) we introduce the following three notions of a transfer function. To avoid confusion, we give different names to the different definitions of transfer functions.

Definition 1.1. For the system (1) we introduce

1. For $\lambda \in \mathbb{C}$ we define the characteristic function $\mathfrak{G}$ in $\lambda$ such that the input-state-output triple $\left(\exp (\lambda t) u_{0}, \exp (\lambda t) x_{0}, \mathfrak{G}(\lambda) u_{0} \exp (\lambda t)\right)$ is a solution of (1) for some $x_{0}$.

2. The input-output transfer function is defined as the Laplace transform of the impulse response, $h(t)=C T(t) B+D \delta(t)$ on its region of convergence $\mathbb{C}_{\beta}^{+}$. 
3. For $\lambda \in \mathbb{C}$ we define an element $y_{\lambda} \in Y$ to be a transmission value for $u_{0} \exp (\lambda t)$ if for the input $u_{0} \exp (\lambda t), t \geq 0$, there exists an initial condition $x_{\lambda}(0) \in X$ such that the output of (1) equals $y_{\lambda} \exp (\lambda t)$ for $t \geq 0$. We say that $G(\lambda)$ is a transmission function at $\lambda$ if for every $u_{0} \in U y_{\lambda}$ can be written as $y_{\lambda}=G(\lambda) u_{0}$.

It is not hard to show that for $s \in \rho(A)$, the resolvent set of $A$,

$$
\mathfrak{G}(s)=C(s I-A)^{-1} B+D,
$$

Furthermore, on the right-half plane $\mathbb{C}_{\omega}^{+}:=\{s \in \mathbb{C} \mid \operatorname{Re}(s)>\omega\}$, we have that $\mathfrak{G}(s)=H(s)=G(s)$, where $\omega$ is the growth bound of $T(t)$.

However, outside this right-half plane things are not so nice. First of all, the transmission function can be multi-valued as is shown in the following example.

Example 1.2. Let $A$ be the left shift on $\ell_{2}(\mathbb{Z})$. So

$$
(A z)_{k}=z_{k+1}, \quad k \in \mathbb{Z} .
$$

We have that

$$
\sigma(A)=\sigma_{c}(A)=\{s \in \mathbb{C}:|s|=1\}
$$

We define $B$ as the vector in $\ell_{2}(\mathbb{Z})$ having all coefficients zero except the coefficient at the position -1 which is taken to be 1 , i.e., $B_{k}=\delta_{-1, k}$. The output operator $C$ is defined as $C z=z_{0}$ and $D=0$. The growth bound of the semigroup generated by $A$ is one, and a simple calculation shows that if $|\lambda|>1$, then

$$
\left((\lambda I-A)^{-1} B\right)_{k}=\lambda^{k} \text { for } k<0 \text {; otherwise } 0 .
$$

On the other hand, if $|\lambda|<1$, then

$$
\left((\lambda I-A)^{-1} B\right)_{k}=-\lambda^{k} \text { for } k \geq 0 ; \text { otherwise } 0 .
$$

So the characteristic function $\mathfrak{G}(\lambda)=C(\lambda I-A)^{-1} B=-1$ if $|\lambda|<1$, but otherwise it is zero.

Let $\lambda \in \rho(A)$ and choose $x_{\lambda}(0)$ in $(1)$ as $p(\lambda)(\lambda I-A)^{-1} B$, where $p(\lambda)$ is an arbitrary constant, then we find that the Laplace transform of $y(t)$ equals

$$
Y_{\lambda}(s)=C(s I-A)^{-1}(\lambda I-A)^{-1} B p(\lambda)+0=p(\lambda) \frac{1}{s-\lambda} C(\lambda I-A)^{-1} B .
$$

Thus for $|\lambda|>1$ this is zero, but for $|\lambda|<1$, we find that any multiple, $p(\lambda)$, can be a transmission value for $u_{0} \exp (\lambda t)$. So we find that the transmission function and value are only unique on some region of the complex plane. 
It can be shown that the transmission values are unique for every $\lambda$ in the resolvent set if the resolvent set consists out of one component. In this case, the transmission function equals the characteristic function. However, even if the resolvent consist of one component, these function need not to be equal to the input-output transfer functions, as is shown in the next section.

\section{Relation between the input-output transfer func- tion and the characteristic function}

In this section, we show that the input-output transfer function need not be a transmission function, even when the transmission function is unique. But first we need to discuss the following simple example.

Example 2.1. Consider the differential equation on $[-1,1]$

$$
\frac{\partial}{\partial t} x(\xi, t)=i \xi x(\xi, t)+\left(1-\xi^{2}\right)^{-1 / 4} u(t)
$$

As state space we choose $L^{2}(-1,1)$. The system operator $A_{0}$ is given by

$$
A_{0} \phi(\xi)=(i \xi) \cdot \phi(\xi)
$$

and this is a bounded operator, with bound 1 , on $L^{2}(-1,1)$. Since it is a multiplication operator, it is easy to see that its spectrum equals $\sigma\left(A_{0}\right)=$ $\{s \in \mathbb{C} \mid \operatorname{Re}(s)=0$ and $|\operatorname{Im}(s)| \leq 1\}$. The input operator $B_{0}$ is defined as $B_{0} u=\left(1-\xi^{2}\right)^{-1 / 4} u$, and since $\left(1-\xi^{2}\right)^{-1 / 4} \in L^{2}(-1,1)$, we know that $B_{0}$ is a bounded operator from $\mathbb{C}$ to $L^{2}(-1,1)$. As output operator, we take the dual of $B_{0}$. Thus

$$
y(t)=\int_{-1}^{1} x(\xi, t)\left(1-\xi^{2}\right)^{-1 / 4} d \xi
$$

The characteristic function of this system is given by the expression

$$
\mathfrak{G}_{0}(s)=\int_{-1}^{1}\left(1-\xi^{2}\right)^{-1 / 2} \frac{1}{(s-i \xi)} d \xi=\frac{\pi}{\sqrt{s^{2}+1}} .
$$

Note that for $s>0$ we have to take the usual (positive) square root of $s^{2}+1$. Thus $\mathfrak{G}_{0}(1 / 2)=2 \pi / \sqrt{5}$. For $s<0$ we have that $\mathfrak{G}_{0}(s)=-\mathfrak{G}_{0}(-s)$, see $(5)$, e.g. $\mathfrak{G}_{0}(-1 / 2)=-2 \pi / \sqrt{5}$.

Concluding we have that the characteristic function is the (unique) analytic continuation of $\pi / \sqrt{s^{2}+1}$ to the resolvent set of $A$, i.e., every complex $s$ except for the interval $[-i, i]$ on the imaginary axis. Since the resolvent set is connected, we have that the characteristic function and the transmission function are the same on the whole resolvent set. 
Example 2.2. Consider the differential equation on $[-1,1]$

$$
\frac{\partial}{\partial t} x(\xi, t)=\frac{1+i \xi}{1-i \xi} x(\xi, t)+(1-i \xi)^{-1}\left(1-\xi^{2}\right)^{-1 / 4} u(t) .
$$

As state space we have again $L^{2}(-1,1)$. The system operator $A_{1}$ is

$$
A_{1} \phi(\xi)=\frac{1+i \xi}{1-i \xi} \cdot \phi(\xi)
$$

and this is a bounded operator, with bound one, on $L^{2}(-1,1)$. Since it is a multiplication operator, it is easy to see that its spectrum equals $\sigma\left(A_{1}\right)=$ $\{s \in \mathbb{C} \mid \operatorname{Re}(s) \geq 0$ and $|s|=1\}$. The input operator $B_{1}$ is defined as $B_{1} u=(1-i \xi)^{-1}\left(1-\xi^{2}\right)^{-1 / 4} u$, and since $(1-i \xi)^{-1}\left(1-\xi^{2}\right)^{-1 / 4} \in L^{2}(-1,1)$, we know that $B_{1} \in \mathcal{L}\left(\mathbb{C}, L^{2}(-1,1)\right)$. As output equation we take

$$
y(t)=2 \int_{-1}^{1} x(\xi, t)(1-i \xi)^{-1}\left(1-\xi^{2}\right)^{-1 / 4} d \xi+\frac{\pi}{\sqrt{2}} u(t) .
$$

Thus the system has a feed-through operator equal to $D_{1}:=\pi / \sqrt{2}$ and an output operator equal to $C_{1} \phi=2 \int_{-1}^{1} \phi(\xi)(1-i \xi)^{-1}\left(1-\xi^{2}\right)^{-1 / 4} d \xi$. For the calculation of the characteristic function we need we the following relations between the system in Example 2.1 and the system defined above.

$$
A_{1}=\left(I+A_{0}\right)\left(I-A_{0}\right)^{-1} \quad B_{1}=\left(I-A_{0}\right)^{-1} B_{0}, \quad C_{1}=2 C_{0}\left(I-A_{0}\right)^{-1} .
$$

Furthermore, $D_{1}=\mathfrak{G}_{0}(1)$. Using this it is not hard to show that

$$
\mathfrak{G}_{1}(s)=\mathfrak{G}_{0}\left(\frac{s-1}{s+1}\right)=\frac{\pi}{\sqrt{\left(\frac{s-1}{s+1}\right)^{2}+1}}=\frac{\pi}{\sqrt{\frac{2 s^{2}+2}{(s+1)^{2}}}} \quad s \neq-1 .
$$

We may simplify the above expression, but extra care should be taken. Let us first define on the set $\rho\left(A_{1}\right)$ the function $f(s):=\frac{\pi}{\sqrt{s^{2}+1}}$. Thus we have taken the branch cut of the square root of $s^{2}+1$ equal to the positive half circle. This is possible, see $[2$, Part II, $\S 12]$. Now define on $\rho\left(A_{1}\right)$ the function

$$
\mathfrak{G}_{2}(s)=\frac{1}{\sqrt{2}}(s+1) f(s) .
$$

Then this function is analytic on $\rho\left(A_{1}\right)$ and it equals $\mathfrak{G}_{1}(s)$ for $s \in(1, \infty)$. This last follows since for $\tilde{s}>0$, one has to take the positive square root in 
$\mathfrak{G}_{0}(\tilde{s})$, see (8). By the uniqueness of an analytic function on a given domain, we conclude that $\mathfrak{G}_{2}(s)=\mathfrak{G}_{1}(s)$ on $\rho\left(A_{1}\right)$. Or equivalently,

$$
\mathfrak{G}_{1}(s)=\frac{\pi}{\sqrt{2}} \frac{s+1}{\sqrt{s^{2}+1}} \quad \text { on } \rho\left(A_{1}\right) .
$$

Please note that $\mathfrak{G}_{1}(s) \neq \frac{1}{\sqrt{2}}(s+1) \mathfrak{G}_{0}(s)$, since they have a different domain. Direct calculations gives that $\mathfrak{G}_{1}(1 / 2)=\mathfrak{G}_{0}(-1 / 3)=-3 \pi / \sqrt{10}$, whereas $\frac{1}{\sqrt{2}}(1 / 2+1) \mathfrak{G}_{0}(1 / 2)=3 \pi / \sqrt{10}$.

Next we derive the impulse response. We know that the impulse response is the inverse Laplace transform of the input-output transfer function. Unfortunately, we don't know this input-output transfer function, since we don't know its region of convergence. However, one does not need its precise region of convergence for the calculation of the inverse Laplace transform. Knowing the input-output transfer function on some right-half plane is sufficient for obtaining its inverse Laplace transform. In this example the growth bound of the semigroup is one, and thus on $\mathbb{C}_{1}^{+}$, we know that the input-output transfer function equals the characteristic function. Thus we must calculate the inverse Laplace transform of $\frac{\pi(s+1)}{\sqrt{2\left(s^{2}+1\right)}}$. We find that this equals

$$
h_{1}(t)=\frac{\pi}{\sqrt{2}}\left[J_{0}(t)-J_{1}(t)+\delta(t)\right],
$$

where $J_{0}$ and $J_{1}$ denote the Bessel functions of the first kind and of the zeroth and first order, respectively.

Since the absolute values of $J_{0}(t)$ and $J_{1}(t)$ decay like $1 / \sqrt{t},[3, \S 7.1]$, we see that the Laplace transform of $h_{1}(t)$ is analytic in the open right-half plane $\mathbb{C}_{0}^{+}$. However, this Laplace transform only equals the transmission function on the smaller right-half plane $\mathbb{C}_{1}^{+}$. It is not hard to see that the Laplace transform of $h_{1}(t)$ equals $H_{1}(s)=\frac{1}{\sqrt{2}}(s+1) \mathfrak{G}_{0}(s)$ on $\mathbb{C}_{0}^{+}$.

1. R.F. Curtain and H.J. Zwart. An Introduction to Infinite-Dimensional Linear Systems Theory. Springer-Verlag, New York, 1995.

2. K. Knopp, Theory of Functions, Part I and II, Two volumes bound as one, Dover Publications, Inc., Mineola, New York, 1996.

3. G.N. Watson, A Treatise on the Theory of Bessel Functions, second edition, Cambridge at the university Press, 1962. 